\title{
Acute coronary syndrome in cancer patients. Part I: pathophysiology, clinical presentation and diagnosis
}

\section{Grzegorz Piotrowski}

Department of Cardio-Oncology,

Medical University of Lodz, Poland

Department of Cardiology,

Provincial Multidisciplinary Oncology and Traumatology Center Nicolaus Copernicus,

Lodz, Poland

Correspondence:

Grzegorz Piotrowski Department of Cardiology, Provincial Multidisciplinary Oncology and Traumatology Center Nicolaus Copernicus, Pabianicka 62,

91-513 Lodz, Poland e-mail:gpiotr4@wp.pl tel.: (+48) 607-244-594 fax: (+48) 42 689-54-91

Received: 15.06.2020 Accepted: 30.06.2020

\section{ABSTRACT}

Coexistence of cardiovascular diseases and cancer is more and more common in everyday practice. This is due to the aging of the population and the fact that most of the classic cardiovascular risk factors also predispose to cancer. During anti-cancer treatment, cardiovascular components may be damaged and eventually lead to acute coronary syndrome. The first part of the review article presents the pathophysiology, clinical presentation and key elements of the diagnostic process of this life-threatening condition in cancer patients.

Key words: cardio-oncology, chemotherapy, acute coronary syndrome, cardiotoxicity, cardiovascular complications 


\section{INTRODUCTION}

It seems that it was long ago when oncology and cardiology were treated as distant, foreign fields that have no common areas of interest with each other. Cardiovascular diseases (CVDs) and cancer are all together responsible for over $2 / 3$ of deaths in the world [1]. The absolute number of people with cancer increases from year to year. This is due to a higher incidence, better detection and increasingly effective anti-cancer treatment.

Due to the enormous progress that has been made in oncology in the last 20 years, patients with cancer have an increasingly better prognosis now. In many cases, recently deadly conditions can be successfully cured nowadays. It is estimated that around 16-17 million people cured of cancer live in the United States (US), which is about $4 \%$ of the total population [2]. Even if there is no complete cure, many months, sometimes long-term remissions are obtained, and cancer becomes chronic, just like most of the CVDs. What is more, many cancers have a better prognosis than some of the CVDs. The best example is chronic heart failure (CHF), in which 5-year survival is significantly worse when compared to the survival in breast cancer in women or prostate cancer and colorectal cancer in men [3].

However, high effectiveness of novel cancer therapies come at a price, which are side effects. If they appear within the circulatory system, it is called cardiotoxicity. Cardiotoxicity that occurs during treatment may lead to its discontinuation - in this situation we are talking about early cardiotoxicity.

This complication limits the oncological prognosis by preventing completion of therapy. Cardiotoxicity may also develop after successful oncological treatment. Sometimes it occurs in cancer survivors after many years of treatment completion, and limits overall survival and worsens quality of life. What is important, cardiotoxicity can affect every element of the circulatory system, including coronary arteries [4].

Coexistence of CVDs and cancer is more and more common in everyday practice. This is due to the aging of the population (age is a risk factor for both diseases) and the fact that most of the classic cardiovascular risk factors also predispose to cancer [5-7]. During effective oncological treatment, components of the cardiovascular system may be damaged and ultimately generate CVD.

The presence of two potentially fatal diseases in one patient entails many diagnostic and therapeutic difficulties and is the cause of poor prognosis. Prediction depends on the simultaneous op- timal treatment of both cancer and CVD [8]. For this reason, the presented review attempts to systematize knowledge about the coexistence of active cancer and acute coronary syndrome (ACS) from the perspective of a practicing cardiologist.

There is no precise data on the coexistence of both the ACS and cancer. It is estimated that cancer in different stages is present in $15 \%$ of patients hospitalized due to ACS [9]. Absolute numbers and percentages of patients with ACS and cancer in hospital statistics are increasing every year. In a retrospective analysis of American data from the National Inpatient Sample (NIS) - the largest database in the US recording hospitalizations, patients with current cancer constituted 1.8\%, while after successful oncological treatment $5.8 \%$ of the population of patients who underwent coronary angioplasty [10]. A distinction should be made between patients with active cancer during oncological therapy and/or diagnostic process and cancer survivors. The nature of the disorders and problems is different in both groups. The discussed issues in the presented study mainly concern patients with active cancer.

Cancer affects all tissues of the body and along with oncological treatment influences the heart muscle and vessels, including coronary arteries. At the time of diagnosis of the proliferative process, but before starting any therapy, approximately $50 \%$ of patients have elevated levels of highly sensitive cardiac troponins and natriuretic peptides [11]. This phenomenon indicates the adverse effects of cancerous tissues on the heart, which releases markers indicating damage and its overloading.

On the one hand, oncological treatment generates cardiovascular damage, on the other hand, it has been noticed that chronic cardiological diseases predispose to proliferative processes and cancer. It seems that cancer develops more frequently in patients with CHF [12]. Recent observations indicate that even after a myocardial infarction, the risk of developing cancer increases [13]. These observations strongly suggest involvement of the same risk factors and similar pathophysiological mechanisms in the development of both CVDs, including atherosclerosis (also coronary artery disease) and cancer.

\section{PATHOPHYSIOLOGY OF ACUTE CORONARY SYNDROME COEXISTING WITH CANCER}

Common risk factors of CVDs and cardiotoxicity of oncological therapy, including the crucial role of inflammation and hypercoagulability, are responsible for frequent coexistence of the coronary artery disease (CAD) and ACS with cancer. Many patients have asymptomatic CAD with often subcritical coronary artery 
changes. They may become symptomatic as a result of inflammation and coagulation activation caused by cancer $[14,15]$. The increasing incidence of ACS and cancer is shown clearly in the statistics. The extending survival of the whole population increases the risk of developing both conditions. There is also increasing evidence that classic CVD risk factors are common to heart and cancer diseases. This mainly applies to such risk factors as smoking, obesity, sedentary lifestyle and hypercholesterolemia [16] Predisposing gene mutations have also been identified both for the development of cancer and for atherosclerotic diseases, including myocardial infarction $[17,18]$. In patients with endothelial dysfunction, which is an early stage of atherosclerosis cancers are more common [19].

Both active cancer and oncological treatment affect the function of the cardiovascular system in a variety of mechanisms. These conditions cause endothelial dysfunction, activation of apoptosis, dysfunction of tunica media, predispose to a vasospasm reaction and activation of the coagulation system (including platelets). They also change the composition of plasma proteins and the expression of adhesion molecules responsible for proper communication of the vessel wall with blood elements, by "unsealing" the endothelium and thus facilitating penetration into the vessel walls by lipoprotein molecules, activated macrophages and other inflammatory cells. All these phenomena lead to accelerated atherosclerosis and finally to the destabilization of the atherosclerotic plaque until its rupture or ulceration (due to apoptosis of the endothelial cells that cover it). Additionally interference of neoangiogenesis within the atherosclerotic plaque and endothelial strokes can lead to its instability [20-22].

Noteworthy is the fact of vascular toxicity (so called vasotoxicity) caused by oncological therapy. Both classic and so-called "new generation" drugs - e.g. targeted molecular therapy, can have a toxic effect on the vessels, including coronary arteries.

From the practical point of view two types of vasotoxicity could be distinguished: irreversible vasotoxicity - type1 and reversible - type 2 .

Type 1 vasotoxicity causes organic changes that lead to narrowing lumen of the arteries. The mechanism of reversible, type 2 vasotoxicity is based on the drug-induced arterial spasm (vasospasm reaction). In this situation after discontinuation of the drug activity, the "toxic" effect withdraws.

Drugs causing dilation of the arteries (nitrates, calcium channel inhibitors) can accelerate dilation or even prevent the va- sospasm. The practical division makes it easy to diagnose and choose the best therapeutic option. Cardiologist assessing the patient with ACS symptoms should consider used oncological drugs in the diagnostic and decision-making process. In the situation where drugs responsible for the type 2 vasotoxicity were used, the drugs should be withdrawn, vasodilating drugs should be included in treatment, and only in the next stages an invasive diagnostics and treatment could be considered.

In turn, from the pathophysiological point of view, vascular toxicity can be divided into three types: A, B and C. Type A - in which the main role in pathophysiology is advanced atherosclerosis and the growth of vasoconstrictive plaques. Type B - defined by the activation of the coagulation pathway leading to clot formation in arteries. Type $\mathrm{C}$ - reversible vasospasm. Thus type $\mathrm{C}$ is equivalent to the type 2 vasospasm reaction outlined above [23].

Type A vascular toxicity can be found after the use of bcr-abl kinase inhibitors (Philadelphia mutation) that revolutionized treatment leukemia with this type of mutation. There are three generations of drugs in this group. The first generation is represented by imatinib, the second by bosutinib, dasatinib, nilotinib and the third by ponatinib. The latter is particularly responsible for the vasotoxic effects. Data from clinical registers and retrospective analysis of medical databases, reveal that the risk of cardiovascular incidences (cerebral, coronary, peripheral) for ponatinib ranged from $9 \%$ to $42 \%$ [24-26]. Vascular incidences when using these drugs may occur in people without prior atherosclerotic disease, but they are thought to be more likely if a patient already had atherosclerosis before. Cardiovascular events can simultaneously affect different vascular areas in one patient. These drugs, by enhancing or accelerating the formation of atherosclerotic plaques, can also give distant vascular consequences manifesting months and even years after completion of anti-cancer treatment [27]. The exact molecular mechanism responsible for vascular complications is unknown. Blockage of abl kinase activity, which plays an important role in vascular endothelial metabolism, imparies the neoangiogenesis through disrupting the function of endothelium of healthy vessels [28]. The therapeutic procedures in type A toxicity during the ACS include evaluation of distinctions of oncological patients and strict control of risk factors, antiplatelet therapy, high doses of statins, an angiotensin converting enzyme inhibitor (ACE-I)/angiotensin II receptor antagonistsl (ARA) and $\beta$-blockers (nebivolol is recommended in this situation).

In type B cardiotoxicity, the main mechanism of causing development of ACS is formation thrombi initiated by used oncological 
therapy. Cisplatin and inhibitors of vascular endothelial growth factor (VEGF) called angiogenesis inhibitors are the main agents in this group. After using cisplatin and its derivatives an acute, even multivessel thrombosis was described [29, 30]. Cisplatin damages arterial endothelium, causing ulcers and erosions, exposing substances activating the coagulation system, which leads to acute arterial thrombosis [31, 32]. Also in long-term observations, cisplatin treatment increases the risk of coronary artery disease and myocardial infarction by 1.5 to 7 times after 10 years. Most often, coronary problems are found in men treated or cured of testicular cancer [33]. The presence of classical risk factors increases the likelihood of a vascular incident.

VEGF inhibitors inhibit the formation of new tumor vessels through impairment of endothelial function. Unfortunately, these drugs also impair the endothelial function of healthy vessels, causing damage, leading to increased thrombosis and vascular incidents [34]. Drugs that belong to this group are: VEGF receptor inhibitor - bevacizumab and small molecule tyrosine kinase inhibitors, which are components of VEGF-associated signaling pathways. This second group includes drugs such as sunitinib, sorafenib or pazopanib. Blockage of the extracellular survival signaling pathways by bevacizumab disturbs the balance of protective and survival mechanisms of the cells to a lesser extent than the intracellular one (small molecule kinase inhibitors tyrosine). Hypertension is the most common side effect of angiogenesis inhibitors [35]. However, blockage of the VEGF signaling pathways leads to dysfunction of endothelium, also at the level of microcirculation, reduction of the synthesis of nitric oxide in favour for constricting factors, including endothelin-1 over vasodilatation substances including nitric oxide [36, 37]. Experimental studies indicate impaired pericyte function and regulation of myocyte contraction in the arterial wall, which may also be part of the pathophysiology of vascular incidents, including ACS [38-40]. Long-term observations suggest that VEGF inhibitors accelerate the growth of atherosclerotic plaques, but do not cause their destabilization. Probably, by inhibiting the neoangiogenesis of vessels in atherosclerotic plaques they can contribute to their stabilization [34]. Management of ACS in type B cardiotoxicity is based on the standard qualification for invasive treatment, the use of dual antiplatelet therapy (the duration of its use is still controversial), statins, ACE-I/ARA and $\beta$-blockers.

Type $C$ of vascular toxicity associated with vasospasm of coronary arteries response most commonly occurs with 5-fluorouracil (5-FU) or its oral derivatives, such as capecitabine. Both drugs are often used to treat colorectal cancer. The incidence of coronary ischemic events with 5 -FU varies from $0,1 \%$ to $20 \%$ [41], twice as rare for capecitabine $[42,43]$. Symptoms of ischemia usually occur shortly after the infusion of the 5-FU. It is manifested by angina pain and ischemic ECG changes (ST elevation/decrease). Prolonged vasoconstriction may lead to myocardial necrosis or generate malignant arrhythmias (ventricular tachycardia/ ventricular fibrillation). Also sudden cardiac deaths have been reported in this population of patients [44]. It seems that the vasoconstrictor response does not depend on endothelial dysfunction but on the disruption of normal regulatory mechanisms of vascular tone [45]. According to this theory, there are premises for the use of vasodilator nitrates and calcium channel blockers therapeutically and preventively before vasospasm. It is believed that the presence of atherosclerotic lesions in the coronary arteries promotes vasoconstrictor response $[42,43]$. After stopping the 5-FU infusion, discontinuation of capecitabine and administration of vasodilators clinical symptoms usually disappear. In such cases, it should be considered whether indications for invasive diagnostics are justified. Release of myocardial necrosis markers (e.g. cardiac troponins) is often observed. The occurrence of cardiotoxicity usually disqualifies the patient from resumption of 5-FU/capecitabine. If there are no alternative therapies, the reuse of these drugs must be done with close monitoring of the electrocardiography (ECG), using smaller, divided doses [46, 47]. Type $C$ vasospastic, reversible toxicity may be caused by taxanes, less often by bleomycin, gemcitabine and vinca alkaloids. The molecular mechanisms of vascular toxicity of these drugs are poorly understood [23].

Another mechanism responsible for cardiac ischemia that should be considered in an oncological patient is tumor compression or neoplastic infiltration of the coronary arteries. Mediastinal tumors and lung cancer can compress or infiltrate the anterior interventricular branch of the left coronary artery. Some lymphomas, e.g. diffuse large B-cell lymphoma (DLBCL) and thymoma may occupy the right ventricle and right coronary artery [48]. Similarly, coronary artery involvement by pericardial mesothelioma or myocardial sarcoma should be considered. Atrial tumors can also compress coronary arteries.

\section{TYPE 1 AND TYPE 2 MYOCARDIAL INFARCTION IN CANCER PATIENTS}

There are 5 types of myocardial infarction (MI) due to the ischemic mechanism [49]. The ischemic mechanism of type $1 \mathrm{Ml}$ is the generation of an unstable atherosclerotic plaque due to its rupture or erosion and acute thrombus formation that closes or significantly restricts coronary artery flow. Cancer predisposes to destabilization of the atherosclerotic plaque (intensification 
of inflammatory processes) and intravascular coagulation. The tumor itself and oncological drugs activate inflammatory and thrombotic pathways and damage the endothelium in various mechanisms. In type $1 \mathrm{Ml}$, the primary goal is to clear the closed or significantly narrowed coronary artery as soon as possible.

In type $2 \mathrm{Ml}$, the balance between myocardial demand and supply of oxygen is disturbed, and this is not a consequence of coronary artery thrombus formation. Even without changes in the arteries, oxygen deficiency can occur due to tachycardia, hypotonia, anemia, hypoxia as a result of respiratory failure (e.g. in lung cancer), infection, sepsis or vasospasm. Patients with cancer often develop these disorders and they are the main mechanisms that lead to ischemia. Atherosclerosis may be present in the artery with type $2 \mathrm{Ml}$, but it is usually negligible and its presence lowers the threshold at which oxygen deficiency occurs, but it is not the leading ischemic disorder. Type $2 \mathrm{Ml}$ is a common phenomenon in cancer patients.

Most of the knowledge about the treatment of $\mathrm{Ml}$ is focused on his type $1 \mathrm{Ml}$. In type $2 \mathrm{Ml}$, the primary actions must be directed primarily at the causes that led to the oxygen deficit in myocardium. If coronary artery changes are suspected, coronary angiography should be performed after correcting the disorders that are the direct mechanism of ischemia, stabilizing the coronary and circulatory system, correcting metabolic, hematologic and hydration abnormalities. Then, if coronary angiography and angioplasty are necessary, they will have a lower risk of complications. Type $2 \mathrm{Ml}$ in the cancer-free population has a worse prognosis than type 1 [50]. There is also no data from clinical trials about what is the optimal treatment in type $2 \mathrm{MI}$. The question of the validity of antiplatelet and anticoagulant treatment remains open if the thrombus was not involved in the ischemic mechanism and the patient simultaneously has thrombocytopenia and a very high risk of bleeding.

\section{PROPOSITION OF DIAGNOSTIC SCHEME FOR CANCER PATIENT WITH SYMPTOMS OF ACUTE CORONARY SYNDROME}

The first step in a diagnostic algorithm in this clinical situation should be to determine the most likely mechanism of ischemia. The following should be always considered: type of cancer, type of oncological therapy, current metabolic and hemodynamic disorders and comorbidities. Differential diagnosis is important, in which it is necessary to consider: pulmonary embolism, pericarditis and non-cardiac diseases such as gastroesophageal reflux disease, pleuritis, pneumothorax. All these conditions can often occur in cancer patients. The following ischemic background symptoms should be considered: 1. reversible mechanism - vasospasm; 2. irreversible - structural - on the basis of atherosclerotic plaque and thrombosis; 3 . mechanical compression of the coronary artery by a tumor. In case of doubt, the first intervention strategy may be to administer vasodilators: intravenous nitroglycerin and calcium channel blockers. Imaging tests will be helpful in diagnostics, and in justified cases diagnostic coronary angiography with the intention of revascularization [23].

\section{CLINICAL PICTURE AND DIFFICULTIES IN DIAGNOSING ACUTE CORONARY SYNDROME IN CANCER PATIENT}

The most pathognomonic symptom of ACS is chest pain with typical presentation. However, coronary pain is less common as a first symptom in the population of cancer patients. Cancer patients more often report dyspnoea, and if the pain occurs it is atypical [51]. Cancer patients take strong analgesics that endure the perception of pain and its threshold. In turn, radiotherapy and chemotherapy can lead to destruction of receptors and the pathways responsible for pain sensation so the threshold sensation is elevated. A common symptom of myocardial infarction in patients with active cancer is hypotension [52].

Electrocardiography (ECG) is another element of routine diagnosis of ACS. Unfortunately, there are no pathognomonic ECG symptoms of heart damage caused by cardiotoxic drugs (anthracyclines, classic cytostatics or immunotherapy). They can cause ECG changes that mimic acute ischemia (ST-T changes, q waves). Nowadays, increased concentrations of cardiac troponins determine the diagnosis of MI. However, in about $50 \%$ of patients, already at the time of cancer diagnosis, significantly higher values of high-sensitivity troponins are found [11]. Also, many anti-cancer drugs "unseal" or damage cardiomyocytes, causing "leakage" of high-sensitivity troponins and an increase of their concentration in the blood. Therefore, in the biochemical diagnosis of MI in this group of patients it is very important to know the baseline values and the variability of cardiac markers in subsequent, sequential determinations.

The criteria for diagnosis of myocardial infarction should be strictly adhered to according to the current universal definition, according to which $\mathrm{Ml}$ is acute ischemic myocardial injury [49]. It is therefore necessary to demonstrate a sudden, significant increase and/or decrease in high-sensitivity troponin levels and to prove that the damage occurred in an ischemic mechanism. Therefore, in the absence of typical MI symptoms, in the presence 
of elevated (or even fluctuations typical for myocardial damage) levels of cardiac troponins, cardiac imaging tests are needed.

The most accessible, non-invasive method of cardiac imaging is echocardiography, which enables detection of evidence of new myocardial dysfunction caused by ischemia or necrosis. In cases where significant narrowing of the coronary artery by a thrombus is suspected, computerized tomography (CT) coronary angiogram may play an important role $[53,54]$.

\section{CONCLUSION}

Diagnosis of $\mathrm{Ml}$ in a cancer patient is a real challenge because of the often ambiguous and confusing symptoms. Therefore, in the ACS diagnostic process in this patient population, it is necessary to take into account the history of the type of cancer, its stage and type of treatment used.

Summarizing the above, the final diagnosis of ACS and myocardial infarction in a cancer patient can be made only in the context of all clinical symptoms and data from medical history.

\section{References}

1. World Health Organization. Global status report on noncommunicable disease 2010. Geneva, Switzerland 2011. http://apps.who.int/iris/bitstream/ handle/10665/44579/9789240686458_eng.pdf.

2. de Moor JS, Mariotto AB, Parry C et al. Cancer survivors in the United States: prevalence across the survivorship trajectory and implications for care. Cancer Epidemiol Biomarkers Prev. 2013; 22(4): 561-70.

3. Stewart S, MacIntyre K, Hole DJ et al. More 'malignant' than cancer? Five-year survival following a first admission for heart failure. Eur J Heart Fail. 200; 3(3): 315-22.

4. Zamorano JL, Lancellotti P, Rodriguez Muñoz D et al. 2016 ESC Position Paper on cancer treatments and cardiovascular toxicity developed under the auspices of the ESC Committee for Practice Guidelines: The Task Force for cancer treatments and cardiovascular toxicity of the European Society of Cardiology (ESC). Eur Heart J. 2016; 37(36): 2768-801.

5. Dreyer L, Olsen JH. Cancer risk of patients discharged with acute myocardial infarct. Epidemiology. 1998; 9(2): 178-83.

6. Zoller B, Ji J, Sundquist J et al. Risk of coronary heart disease in patients with cancer: a nationwide follow-up study from Sweden. Eur J Cancer. 2012; 48(1): $121-8$.

7. Weaver $\mathrm{KE}$, Foraker $\mathrm{RE}$, Alfano $\mathrm{CM}$ et al. Cardiovascular risk factors among long-term survivors of breast, prostate, colorectal, and gynecologic cancers: a gap in survivorship care? J Cancer Surviv. 2013; 7(2): 253-61.

8. Iliescu CA, Grines CL, Herrmann J et al. SCAI Expert consensus statement: Evaluation, management, and special considerations of cardio-oncology patients in the cardiac catheterization laboratory (endorsed by the cardiological society of India, and sociedad Latino Americana de Cardiologia intervencionista). Catheter Cardiovasc Interv. 2016; 87(5): E202-23.

9. Chen HY, Saczynski JS, McManus DD et al. The impact of cardiac and noncardiac comorbidities on the short-term outcomes of patients hospitalized with acute myocardial infarction: a population-based perspective. Clin Epidemiol. 2013; 5: 439-48.

10. Potts JE, lliescu CA, Lopez Mattei JC et al. Percutaneous coronary intervention in cancer patients: a report on the prevalence and outcomes in the United States. Eur Heart J. 2019; 40: 1790-800.

11. Pavo N, Raderer M, Hülsmann M et al. Cardiovascular biomarkers in patients with cancer and their association with all-cause mortality. Heart. 2015; 101(23): 1874-80.

12. Ameri $P$, Canepa $M$, Anker MS et al. Cancer diagnosis in patients with heart failure: epidemiology, clinical implications and gaps in knowledge. Eur J Heart Fail. 2018; 20(5): 879-87.

13. Malmborg M, Christiansen CB, Schmiegelow MD et al. Incidence of new onset cancer in patients with a myocardial infarction - a nationwide cohort study. BMC Cardiovasc Disord. 2018; 18(1): 198.

14. Demers M, Krause DS, Schatzberg D et al. Cancers predispose neutrophils to release extracellular DNA traps that contribute to cancer-associated thrombosis. Proc Natl Acad Sci U S A. 2012; 109(32): 13076-81.

15. Blann AD, Dunmore S. Arterial and venous thrombosis in cancer patients. Cardiol Res Pract. 2011; 2011:394740.

16. Nelson ER, Wardell SE, Jasper JS et al. 27-Hydroxycholesterol links hypercholesterolemia and breast cancer pathophysiology. Science. 201; 342(6162): 1094-8.

17. Jaiswal S, Natarajan P, Silver AJ et al. Clonal Hematopoiesis and Risk of Atherosclerotic. N Engl J Med. 2017; 377(2): 111-21.

18. Jaiswal S, Natarajan P, Ebert BL. Clonal Hematopoiesis and Atherosclerosis. N Engl J Med. 2017; 377(14): 1401-2.

19. Toya T, Sara JD, Corban MT et al. Assessment of peripheral endothelial function predicts future risk of solid-tumor cancer. Eur J Prev Cardiol. 2019; 27(60): 608-18. http://doi.org/10.1177/2047487319884246.

20. Davignon J, Ganz P. Role of endothelial dysfunction in atherosclerosis. Circulation. 2004; 109(23 suppl 1): III27-III32.

21. Herrmann J, Lerman A. An update on cardio-oncology. Trends Cardiovasc Med. 2014; 24(7): 285-95.

22. Wang JC, Normand SL, Mauri L et al. Coronary artery spatial distribution of acute myocardial infarction occlusions. Circulation. 2004; 110(3): 278-84.

23. Herrmann J. Clinical Cardio-oncology. Elsevier, Phyladelphia 2017: 173.

24. Cortes JE, Kim DW, Pinilla-Ibarz J et al. A phase 2 trial of ponatinib in Philadelphia chromosome-positive leukemias. N Engl J Med. 2013; 369(19): 1783-96.

25. Cortes JE, Kantarjian H, Shah NP et al. Ponatinib in refractory Philadelphia chromosome-positive leukemias. N Engl J Med. 2012; 367(22): $2075-88$.

26. Nicolini FE, Gagnieu MC, Heiblig M et al. Cardio-vascular events occurring on ponatinib in chronic phase chronic myeloid leukemia patients, preliminary analysis of a multicenter cohort. Blood. 2013; 122(21): 4020.

27. Levato L, Cantaffa R, Kropp MG et al. Progressive peripheral arterial occlusive disease and other vascular events during nilotinib therapy in chronic myeloid leukemia: a single institution study. Eur J Haematol. 2013; 90(6): 531-2.

28. Chislock EM, Ring C, Pendergast AM. Abl kinases are required for vascular function, Tie2 expression, and angiopoietin-1-mediated survival. Proc Natl Acad Sci U S A. 2013; 110(30): 12432-7.

29. Berliner S, Rahima M, Sidi Y et al. Acute coronary events following cisplatin-based chemotherapy. Cancer Invest. 1990; 8(6): 583-6.

30. Jafri M, Protheroe A. Cisplatin-associated thrombosis. Anticancer Drugs. 2008; 19(9): 927-9.

31. Karabay KO, Yildiz O, Aytekin V. Multiple coronary thrombi with cisplatin. J Invasive Cardiol. 2014; 26(2): E18-20.

32. Togna Gl, Togna AR, Franconi M et al. Cisplatin triggers platelet activation. Thromb Res. 2000; 99(5): 503-9. 
33. Oh JH, Baum DD, Pham S et al. Long-term complications of platinum-based chemotherapy in testicular cancer survivors. Med Oncol. 2007; 24(2): $175-81$.

34. Winnik S, Lohmann C, Siciliani G et al. Systemic VEGF inhibition accelerates experimental atherosclerosis and disrupts endothelial homeostasis implications for cardiovascular safety. Int J Cardiol. 2013; 168(3): 2453-61.

35. Kappers $\mathrm{MH}$, van Esch JH, Sluiter W et al. Hypertension induced by the tyrosine kinase inhibitor sunitinib is associated with increased circulating endothelin-1 levels. Hypertension. 2010; 56(4): 675-81.

36. Kappers MH, Smedts FM, Horn T et al. The vascular endothelial growth factor receptor inhibitor sunitinib causes a preeclampsia-like syndrome with activation of the endothelin system. Hypertension. 2011; 58(2): 295-302.

37. Kappers $\mathrm{MH}$, de Beer VJ, Zhou Z et al. Sunitinib-induced systemic vasoconstriction in swine is endothelin mediated and does not involve nitric oxide or oxidative stress. Hypertension. 2012; 59(1): 151-7.

38. Chintalgattu V, Rees ML, Culver JC et al. Coronary microvascular pericytes are the cellular target of sunitinib malate-induced cardiotoxicity. Sci Transl Med. 2013; 5(187): 187ra69.

39. de Boer MP, van der Veldt AA, Lankheet NA et al. Sunitinib-induced reduction in skin microvascular density is a reversible phenomenon. Ann Oncol. 2010; 21(9): 1923-4.

40. van der Veldt AA, de Boer MP, Boven E et al. Reduction in skin microvascular density and changes in vessel morphology in patients treated with sunitinib. Anticancer Drugs. 2010; 21(4): 439-46.

41. Südhoff T, Enderle MD, Pahlke $M$ et al. 5-Fluorouracil induces arterial vasocontractions. Ann Oncol. 2004; 15(4): 661-4.

42. Cerny J, Hassan A, Smith C et al. Coronary vasospasm with myocardial stunning in a patient with colon cancer receiving adjuvant chemotherapy with FOLFOX regimen. Clin Colorectal Cancer. 2009; 8(1): 55-8.

43. Ambrosy AP, Kunz PL, Fisher GA et al. Capecitabine-induced chest pain relieved by diltiazem. Am J Cardiol. 2012; 110(11): 1623-6.

44. Stewart T, Pavlakis N, Ward M. Cardiotoxicity with 5-fluorouracil and capecitabine: more than just vasospastic angina. Intern Med J. 2010; 40(4): 303-7.

45. Polk A, Vistisen K, Vaage-Nilsen M et al. A systematic review of the pathophysiology of 5-fluorouracil-induced cardiotoxicity. BMC Pharmacol Toxicol. 2014; 15: 47.

46. Jensen SA, Sørensen JB. Risk factors and prevention of cardiotoxicity induced by 5-fluorouracil or capecitabine. Cancer Chemother Pharmacol. 2006; 58(4): 487-93.

47. Polk A, Vistisen K, Vaage-Nilsen M et al. Cardiotoxicity in cancer patients treated with 5-fluorouracil or capecitabine: a systematic review of incidence, manifestations and predisposing factors. Cancer Treat Rev. 2013; 39(8): 974-84.

48. Juan $\mathrm{YH}$, Chatzizisis YS, Saboo SS et al. Tumor encasement of the right coronary artery: role of anatomic and functional imaging in diagnosis and therapeutic management. Open Cardiovasc Med J. 2014; 8: 110-2.

49. Thygesen K, Alpert JS, Jaffe AS et al. [Fourth universal definition of myocardial infarction (2018)]. Kardiol Pol. 2018; 76(10): 1383-415.

50. Singh A, Gupta A, DeFilippis EM et al. Cardiovascular Mortality After Type 1 and Type 2 Myocardial Infarction in Young Adults. J Am Coll Cardiol. 2020; 75(9): 1003-13.

51. Yusuf SW, Daraban N, Abbasi N et al. Treatment and outcomes of acute coronary syndrome in the cancer population. Clin Cardiol. 2012; 35(7): 443-50.

52. Piotrowski G, Pszonicka K, Paradowska E et al. Global Cardio-Oncology Summit. Vancouver 2015.

53. Ibanez B, James S, Agewall S et al. ESC Scientific Document Group. 2017 ESC Guidelines for the management of acute myocardial infarction in patients presenting with ST-segment elevation: The Task Force for the management of acute myocardial infarction in patients presenting with ST-segment elevation of the European Society of Cardiology (ESC). Eur Heart J. 2018; 39(2): 119-77.

54. Roffi M, Patrono C, Collet JP et al. ESC Scientific Document Group. 2015 ESC Guidelines for the management of acute coronary syndromes in patients presenting without persistent ST-segment elevation: Task Force for the Management of Acute Coronary Syndromes in Patients Presenting without Persistent ST-Segment Elevation of the European Society of Cardiology (ESC). Eur Heart J. 2016; 37(3): $267-315$. 